\title{
Melhoria da Qualidade de Produto e de Processo de Software a partir da Análise de Indicadores de Teste
}

\author{
ERIKA DE FREITAS NITA \\ CI\&T SYSTEMS S/A \\ www.cit.com.br
}

\begin{abstract}
Resumo
Atualmente, a maioria das empresas de software enfrenta problemas relacionados à necessidade de melhorar a qualidade do produto e a satisfação do cliente mantendo custo baixo e cumprindo prazos. Este artigo relata a experiência de uma organização que utiliza a análise dos indicadores de teste como ferramenta para a melhoria do processo de desenvolvimento e a garantia da qualidade do produto.

PALAVRAS-CHAVE: teste, qualidade, produto, processo, medida, métrica, indicador, meta
\end{abstract}

\begin{abstract}
Currently, most of the world wide software companies faces problems related to the need to improve product quality and customer satisfaction while maintaining low costs and meeting deadlines. This paper reports the experience of a software organization which uses test indicator analysis as a tool for process improvement and product quality assurance.

KEY-WORDS: test, quality, product, process, measurement, metric, indicator, goal
\end{abstract}

\section{Introdução}

Atualmente, existe uma crescente preocupação das empresas de software com a satisfação de seus clientes, com a produtividade de suas equipes e com os custos de seus projetos. Todo o esforço na melhoria da qualidade de software está centrado, basicamente, nesses três pontos. Mas, apesar disso, sabe-se que apenas um pequeno percentual de projetos podem ser considerados de sucesso, ou seja, projetos que terminam no prazo, dentro do orçamento e satisfazendo a todos os requisitos previstos. Além disso, muitos projetos são cancelados ou não implantados por grandes atrasos, estouros de orçamento e/ou não disponibilização de todos os requisitos previstos [1].

É nesse contexto que cresce a importância dos testes, medição e gerenciamento dos defeitos e análise do processo de desenvolvimento como formas de se resolver questões sobre o custo (em tempo e esforço) da correção de defeitos ou o efeito de um defeito encontrado em produção na satisfação do cliente.

Este texto descreve a experiência de uma empresa na utilização dos indicadores de teste para melhoria de seu produto e processo de desenvolvimento, tendo como objetivo principal a diminuição de custos, além do ganho de produtividade e aumento da satisfação de seus clientes. Nas seções a seguir, será abordado como foi montada a equipe responsável pelos testes e análise dos indicadores, como foi definida a metodologia de teste, a escolha, obtenção e análise dos indicadores, e a apresentação dos resultados.

\section{Concepção do Processo de Teste}

A atividade de teste sempre fez parte do processo de desenvolvimento de software, porém, na maioria das empresas os testes nem sempre são tratados com a devida seriedade. $\mathrm{O}$ que se 
testemunha são testes feitos muitas vezes pelo próprio analista de requisitos ou implementador, sem planejamento ou análise de cobertura, de forma estanque após o término do desenvolvimento e pouco antes da entrega do produto.

Quando os testes não são realizados por uma equipe independente e não têm um planejamento prévio, acaba-se viciando as verificações executadas na aplicação ou deixando de considerar situações relevantes, não ajudando na identificação e eliminação de inconsistências de especificação ou falhas de implementação.

Também, ao se deixar a realização dos testes apenas para o final do projeto, corre-se o risco de prejudicar a fase de teste por não se ter tempo para verificar a aplicação como planejado e resolver os problemas encontrados, por causa de atrasos durante $\mathrm{o}$ desenvolvimento do software.

\subsection{Formação da Equipe de Teste}

Para se tentar eliminar esses já conhecidos problemas do teste de software, iniciou-se a formação de uma equipe de testes que fosse mais que apenas pessoas que executassem algumas poucas simulações de uso da aplicação pouco antes da entrega do produto ao cliente. Começou-se a formação de uma equipe altamente capacitada.

Foi feito investimento em uma equipe de testes especializada que é capaz, além de encontrar defeitos de implementação, de criticar todos os artefatos produzidos até então, já que a maioria deles servirão de entrada para as atividades de teste. Pessoas capazes de identificar no próprio processo de desenvolvimento, incluindo o processo de teste, falhas que pudessem contribuir para o retrabalho, atrasos, e aparecimento de defeitos em produção.

Foi criada uma equipe autônoma e independente, mas com grande interação com a de desenvolvimento e tão comprometida com o sucesso do projeto como todos os outros da equipe.

\subsection{Definição da Metodologia de Teste}

Além de pessoal altamente capacitado também foi preciso escolher uma metodologia que não tivesse o foco somente na execução de teste e que contribuísse para o processo de desenvolvimento de software da empresa. Uma metodologia que contemplasse um planejamento antecipado e uma maior abrangência, e que cuidasse das situações anormais do uso do sistema.

Outro ponto importante na escolha da metodologia é a possibilidade de se manter a rastreabilidade das informações levantadas durante a execução de testes (como defeitos e sugestões) para se analisar o efeito das alterações originadas por elas.

O processo de teste ficou, de forma geral, dividido nas atividades de Planejamento, Projeto, Execução e Avaliação, como mostrado na Tabela 1.

\begin{tabular}{|l|ll|}
\hline \multicolumn{1}{|c|}{ Atividade } & \multicolumn{1}{c|}{ Descrição } \\
\hline Planejamento de teste & $\circ$ & Participar do levantamento de requisitos \\
& $\circ$ & Identificar os tipos de teste \\
& $\circ$ & Levantar riscos \\
\hline Projeto de teste & $\circ$ & Criação de casos de teste \\
\hline
\end{tabular}




\begin{tabular}{|l|l|}
\hline & $\circ$ Preparação de banco de dados de teste \\
& $\circ$ Preparação de scripts para os testes automatizados \\
\hline Execução dos testes & $\circ$ Aplicação dos casos de teste \\
& $\circ$ Detecção de defeitos \\
& $\circ$ Controle dos defeitos encontrados \\
& $\circ$ Reteste \\
\hline Avaliação dos testes & $\circ$ Medidas associadas aos testes \\
& $\circ$ Métricas de qualidade \\
& $\circ$ Realimentação do processo de desenvolvimento \\
\hline
\end{tabular}

Tabela 1 - Atividades do processo de teste

Os testes foram classificados segundo um critério chamado "Estágio", de acordo com o momento em que seriam realizados. Assim, os testes podem ser unitários, de integração, de sistema ou de aceitação. Cada um desses testes devem ser realizados em momentos bem delimitados e com responsabilidade bem definida para a sua execução, como mostrado na Tabela 2.

\begin{tabular}{|l|l|}
\hline \multicolumn{1}{|c|}{ Estágio de Teste } & \multicolumn{1}{c|}{ Descrição } \\
\hline Testes unitários & $\circ$ Quando: durante a implementação \\
& $\circ$ Como: teste de classes e pedaços de código isolados \\
& $\circ$ Responsável: implementador \\
\hline Testes de integração & $\circ$ Quando: durante a implementação \\
& $\circ \begin{array}{l}\text { Como: teste da integração entre as funcionalidades } \\
\text { desenvolvidas pelos vários implementadores }\end{array}$ \\
& $\circ$ Responsável: integrador \\
\hline Testes de sistema & $\circ$ Quando: ao término do desenvolvimento \\
& $\circ \begin{array}{l}\text { Como: aplicação do planejamento de teste, em ambiente de } \\
\text { teste }\end{array}$ \\
\hline Testes de aceitação & $\circ$ Responsável: analista de teste \\
& $\circ$ Quando: após entrega ao cliente \\
& $\circ$ Como: aplicação de um subconjunto do planejamento de teste, \\
& $\circ$ em ambiente de produção \\
& $\circ$ Responsável: analista de teste \\
\hline
\end{tabular}

Tabela 2 - Estágios de teste segundo o momento de sua execução

O objetivo da adoção dessas atividades e dessa classificação dos testes foi alcançar a diminuição do risco de grandes desvios de custo e prazo, a taxa de retrabalho não previsto, o custo da correção de defeitos, e aumentar a cobertura dos testes. Em última análise, a meta é diminuir ao máximo os defeitos encontrados após a entrada do sistema em produção.

\section{Obtenção dos Indicadores}

Antes de apresentar os indicadores escolhidos, seguem alguns conceitos adotados na empresa: 
× Medida: é a quantificação de um atributo do projeto;

× Medição: é a coleta de uma medida do projeto em um dado instante do tempo;

x Métrica: é a função de dois ou mais valores de medidas;

× Indicador: pode ser uma medida ou métrica. Usado como critério de decisão;

× Meta: é um nível de resultado que deve ser atingido por um determinado indicador no projeto.

Obs.: entende-se por problemas de indicador um valor muito distante da sua meta.

\subsection{Definição de Indicadores}

Para se obter os indicadores que serviriam de base para a melhoria da qualidade do processo, foi preciso primeiro fazer a medição dos projetos. Para isso, foi necessário, antes de tudo, ter definido um conjunto de medidas que trouxessem dados das atividades executadas.

A definição das medidas foi baseada nas atividades realizadas e artefatos produzidos durante o processo de desenvolvimento utilizado na empresa. Foram selecionadas atividades consideradas relevantes de se medir e as métricas que se gostaria de obter [4]. Foi elaborada uma primeira versão com um pequeno conjunto de medidas que seriam coletadas dos projetos.

Após as primeiras análises feitas sobre as medidas extraídas dos projetos, sentiu-se a necessidade de outras medidas que não faziam parte do conjunto de medidas de projeto para complementar linhas de raciocínio e se chegar a algumas conclusões.

Por isso, elaborou-se um segundo conjunto se medidas, um pouco mais complexo mas que dava maior argumentação no momento de se concluir as análises feitas sobre os resultados dos projetos. Existiram, ainda, outros ciclos de melhoria, antes de se chegar ao conjunto de indicadores atual. Essas melhorias sempre são motivadas pela necessidade dos próprios projetos em ter mais informações para analisar os seus resultados.

A Tabela 3 mostra um subconjunto atual dos indicadores de projeto da empresa.

\begin{tabular}{|l|l|}
\hline \multicolumn{1}{|c|}{ Indicador } & \multicolumn{1}{|c|}{ Descrição } \\
\hline Cobertura do planejamento de teste & $\begin{array}{l}\text { percentagem de requisitos cobertos pelo } \\
\text { planejamento de teste }\end{array}$ \\
\hline Cobertura da execução de teste & $\begin{array}{l}\text { percentagem de requisitos cobertos pela execução de } \\
\text { teste }\end{array}$ \\
\hline $\begin{array}{l}\text { Produtividade de planejamento de } \\
\text { teste }\end{array}$ & número médio de casos de teste planejados por hora \\
\hline Produtividade de execução de teste & número médio de casos de teste executados por hora \\
\hline $\begin{array}{l}\text { Esforço de planejamento/projeto de } \\
\text { teste }\end{array}$ & $\begin{array}{l}\text { percentual das horas de planejamento/projeto sobre o } \\
\text { total das horas gastas em teste }\end{array}$ \\
\hline Esforço de execução de teste & $\begin{array}{l}\text { percentual das horas de execução sobre o total das } \\
\text { horas gastas em teste }\end{array}$ \\
\hline $\begin{array}{l}\text { Natureza dos defeitos encontrados } \\
\text { pela equipe de testes }\end{array}$ & $\begin{array}{l}\text { percentual dos defeitos encontrados para cada } \\
\text { natureza de defeitos pré-definida }\end{array}$ \\
\hline $\begin{array}{l}\text { Defeitos encontrados pela equipe de } \\
\text { testes }\end{array}$ & $\begin{array}{l}\text { número de defeitos encontrado durante a fase de } \\
\text { testes, para cada 100.000 linhas de código }\end{array}$ \\
\hline
\end{tabular}




\begin{tabular}{|l|l|}
\hline Defeitos encontrados em produção & $\begin{array}{l}\text { número de defeitos encontrado após a entrada do } \\
\text { software em produção, para cada 100.000 linhas de } \\
\text { código }\end{array}$ \\
\hline Esforço de teste unitário & $\begin{array}{l}\text { percentual das horas de teste unitário sobre o total } \\
\text { das horas gastas em implementação }\end{array}$ \\
\hline Retrabalho de implementação & $\begin{array}{l}\text { percentual de esforço gasto na atividade de correção } \\
\text { de defeitos de implementação }\end{array}$ \\
\hline Desvio de esforço no projeto & $\begin{array}{l}\text { diferença entre o esforço planejado e o esforço } \\
\text { realizado no projeto ou fase/iteração do projeto }\end{array}$ \\
\hline Desvio de custo do projeto & $\begin{array}{l}\text { diferença entre o custo planejado e o custo realizado } \\
\text { no projeto ou fase/iteração do projeto }\end{array}$ \\
\hline Senioridade da equipe & $\begin{array}{l}\text { meses de experiência dos membros da equipe no } \\
\text { desempenho do seu papel no projeto }\end{array}$ \\
\hline Satisfação da equipe & $\begin{array}{l}\text { satisfação dos membros da equipe do projeto quanto } \\
\text { à gestão interna }\end{array}$ \\
\hline Satisfação do cliente & $\begin{array}{l}\text { satisfação dos clientes com os resultados } \\
\text { apresentados no projeto }\end{array}$ \\
\hline Custo dos defeitos & $\begin{array}{l}\text { esforço médio gasto para a correção dos defeitos no } \\
\text { projeto ou fase/iteração do projeto }\end{array}$ \\
\hline
\end{tabular}

Tabela 3 - Indicadores de projeto usados na análise de teste

\subsection{Freqüîncia das Medições}

As medições são realizadas em todos os projetos ao final de cada iteração de cada fase ${ }^{1}$, quando é disparada pela gerência senior de cada projeto, de acordo com seus prazos. É acionada a equipe $\mathrm{SEPG}^{2}$ para obtenção dos indicadores e avaliação do cumprimento das metas, sendo que cada fase tem um conjunto de metas a serem avaliadas.

\section{Avaliação do Processo de Desenvolvimento}

Após a coleta de todas as medidas do projeto é que a equipe de teste tem todo o material necessário para começar o seu trabalho de análise crítica dos resultados. São analisados não só os indicadores de teste, mas alguns outros indicadores do projeto que são identificados como diretamente relacionados com os resultados obtidos.

\subsection{Análise dos Indicadores do Projeto}

Os primeiros indicadores analisados são aqueles que relacionam o esforço de teste com a qualidade do produto entregue ao cliente. Por exemplo:

x relação da produtividade de teste (tanto no planejamento como na execução) com o número de defeitos encontrados em produção;

\footnotetext{
${ }^{1}$ São quatro as fases de um projeto: concepção, elaboração, construção e transição, de acordo com o processo de desenvolvimento seguido na empresa [2].

${ }^{2}$ Software Engineering Process Group: grupo sugerido pelo CMM[3], responsável pelas atividades de processo de software.
} 
× relação da cobertura do planejamento e execução dos teste com o número de defeitos encontrados em produção;

× relação da percentagem de horas de planejamento e execução de teste com o número de defeitos encontrados em produção.

Depois, faz-se uma ligação dos indicadores de teste e do projeto, com os resultados obtidos. Por exemplo:

× relação entre o número de defeitos encontrados pela equipe de testes e a satisfação da equipe com a gerência interna;

x diferença entre o número de defeitos encontrados pela equipe de testes e os encontrados em produção;

× relação entre a percentagem de horas de teste unitário e o número de defeitos encontrados pela equipe de teste;

× relação entre a percentagem de horas de teste unitário e o número de defeitos encontrados em produção;

x relação da produtividade de teste (tanto no planejamento como na execução) com o desvio de esforço do projeto;

x relação entre a taxa de retrabalho de implementação e o desvio de esforço do projeto;

x relação entre o custo dos defeitos e o desvio de custo do projeto;

× relação entre o número de defeitos encontrados em produção e a satisfação do cliente;

× relação do percentual de defeitos de acordo com sua natureza e a senioridade da equipe.

\subsection{Análise dos Indicadores no Processo de Desenvolvimento}

Depois da análise feita sobre a qualidade do produto resultante do projeto, faz-se uma relação desses números com as atividades do processo de desenvolvimento. É possível, entre outras análises, verificar que:

× problemas na produtividade de planejamento dos testes, indicam necessidade de melhorias nas atividades de especificação dos requisitos;

× problemas na produtividade de execução de testes e/ou número excessivo de defeitos encontrados em produção, indicam necessidade de melhorias nas atividades de planejamento de teste;

× problemas com número excessivo de defeitos encontrados em produção, estão relacionados com um grande número de defeitos encontrados pela equipe de testes e podem indicar necessidade de melhorias nas atividades de teste unitário;

x a relação entre o número de defeitos encontrados pela equipe de testes e a satisfação da equipe, pode indicar necessidade de melhorias nas atividades de gerência de projeto;

* a relação entre a taxa de retrabalho de implementação e o desvio de esforço do projeto, pode indicar necessidade de melhorias nas atividades de implementação;

x a relação entre o custo dos defeitos e o desvio de custo do projeto, pode indicar necessidade de melhorias nas atividades de teste; 
* a relação entre a natureza dos defeitos encontrados e a senioridade da equipe, pode indicar necessidade de melhorias nas atividades de gerência de projetos e de planejamento de capacitação de recursos humanos.

\section{Resultados}

Durante o último ano ficaram evidentes os ganhos dessa abordagem do uso de indicadores de teste e seus relacionamentos com outros indicadores dos projeto. Merecem destaque, dentre os resultados positivos alcançados até agora:

x maior comprometimento com os resultados: ficou claro para todos da equipe o significado de cada indicador dentro do projeto, principalmente os relacionados aos defeitos. O assunto "defeitos", antes recebido com resistência pela equipe, agora é abertamente abordado nas reuniões de status, com discussões sobre causas de defeitos, e boas práticas para evitá-los;

× diminuição no número de defeitos: com as iniciativas de melhoria propostas pelo grupo de teste nas atividades de implementação, houve uma queda de mais de $20 \%$ na inserção de defeitos durante a implementação;

× ganho de produtividade: com as iniciativas de melhoria propostas pelo grupo de teste nas suas próprias atividades, houve um ganho de mais $15 \%$ na produtividade de planejamento e execução de testes;

x aumento da satisfação do cliente: com o trabalho feito pelo grupo de testes para diminuir o números de defeitos de implementação, houve um aumento de quase $8 \%$ na satisfação do cliente;

× diminuição dos atrasos de entrega: com o trabalho feito pelo grupo de testes para diminuir a taxa de retrabalho, houve uma queda de $10 \%$ nos desvios de esforço de projeto;

x diminuição dos custos: com o trabalho feito pelo grupo de testes para se encontrar o quanto antes o maior número possível de defeitos, houve uma queda de quase $5 \%$ nos desvios de custo de projeto.

\subsection{Dificuldades}

Para se obter os valores dos indicadores, foi preciso inserir maior disciplina e controle sobre as atividades realizadas. Por isso, foi observada alguma resistência por parte das equipes de projeto. Esse problema foi abordado, primeiramente, com reuniões das equipes de projeto com suas gerências senior para uma melhor explicação sobre a necessidade de se ter maior disciplina na realização e registro das atividades do processo de desenvolvimento. Tentou-se deixar claro que dessa disciplina dependia a precisão das medidas extraídas dos projetos e, consequentemente, a confiabilidade das análises que seriam feitas sobre esses dados.

Após essas reuniões com a gerência senior, também foram criados grupos de trabalho para se tentar eliminar por completo as resistências que ainda existiam. Formou-se um grupo de estudo para cada um dos papéis das atividades mais críticas nos projetos, como analistas de requisitos, gerentes de projeto e o próprio analistas de testes. Esses grupos são formados por pessoas que desempenham tais papéis nos projetos e elas se reúnem pelo menos uma vez por mês para discutir, entre outros tópicos, suas metas e os resultados dos indicadores relacionados às suas atividades. Nesse ano de 2002, foram esses grupos que determinaram 
quais seriam os indicadores de qualidade e produtividade dos projetos, e suas respectivas metas.

Outra dificuldade está em manter atualizadas as informações sobre as medidas de projeto. Está sendo necessário um esforço extra para se conquistar o compromisso da gerência de projeto em se manter a regularidade das coletas de medidas nos projetos.

\subsection{Desafios}

Apesar dos bons resultados obtidos até agora, ainda existe muito o que fazer. Os pontos mais urgentes a serem melhorados são:

x ampliar os serviços de SQA (segundo o modelo CMM), como serviços de inspeção de artefatos e auditoria no processo, para $100 \%$ dos projetos (hoje apenas os projetos mais críticos têm esses serviços) a fim de levantar os "defeitos" não só de implementação, mas de todos os outros fluxos de atividades de um projeto;

× implantar a medida de pontos por função segundo o IFPUG ${ }^{3}$ para se poder comparar os resultados da empresa com as metas estabelecidas pelo mercado. Atualmente, utiliza-se uma medida de tamanho funcional com metodologia de contagem proprietária e não universal.

\section{Conclusão}

A nova visão da qualidade de software está voltada para prevenção de defeitos através da melhoria do processo de desenvolvimento de software. Essa nova abordagem deixa de lado a idéia de que o teste de software é mero coadjuvante no processo de desenvolvimento.

É fundamental a definição de um processo de desenvolvimento, incluindo o de teste, para que seja possível a extração de medidas das atividades realizadas e artefatos produzidos nos projetos. Somente dessa maneira será possível a obtenção dos indicadores que, juntamente com uma equipe de testes independente e capacitada, possibilitarão a análise e comparação dos resultados dos projetos da empresa.

Assim, estará garantida a melhoria do processo de desenvolvimento e, consequentemente, a melhoria da qualidade do produto entregue ao cliente.

\section{Referências}

[1] The Standish Group International, Inc.. The CHAOS Report. 1995.

[2] Jacobson, I.; Booch, G.;Rumbaugh, J. The Unified Software Development Process, Addison-Wesley, 1999.

[3] Paulk, M.; Curtis, B.; Chrissis, M.; Weber, C. Capability Maturity Model for Software, Version 1.1. Technical Report - CMU/SEI-93-TR-24, Software Engineering Institute, Carnegie Mellon, February 1993.

[4] Kan, Stephen H. Metrics and Models in Software Quality Engineering, Addison-Wesley, 1994.

\footnotetext{
${ }^{3}$ International Function Point Users Group: http://www.ifpug.org/publications/manual.htm
} 